\title{
De la empresa al mercado: factores que inciden en la organización de las cadenas de producción de bioenergía*
}

\section{From the business to the market: factors that affect \\ the organization of bioenergy production chains}

María Eugenia Castelao Caruana**

Recibido: 16 de diciembre de 2016

Revisado: 16 de enero de 2017

Aprobado: 3 de febrero de 2017

\section{Resumen}

Aunque existen importantes oportunidades para el desarrollo de la industria de la bioenergía en América Latina, la industria de la bioenergía en materia de generación eléctrica está sumamente rezagada. La literatura internacional coincide en destacar la importancia de las barreras económicas, sociales e institucionales en la penetración de

* Trabajo realizado en el marco del proyecto "Cooperativas de provisión eléctrica: factores limitantes para el desarrollo de proyectos bioenergéticos en las regiones Centro y Pampeana de Argentina", financiado por el Consejo Nacional de Investigaciones Científicas y Técnicas. ORCID ID del autor: orcid.org/0000-0003-0181-9862

** Doctora en Ciencias Económicas, investigadora asistente en la Universidad de Buenos Aires - Consejo Nacional de Investigaciones Científicas y Técnicas. Facultad de Ciencias Económicas. Buenos Aires, Argentina. Correo electrónico: eugecastelao@gmail.com 
esta industria en el mercado de la energía eléctrica. Para abordar estos factores, los proyectos de bioenergía a nivel individual desarrollan diversas formas de coordinación de los recursos a lo largo de la cadena de producción. Este trabajo presenta los resultados de una investigación exploratoria sobre las barreras que condicionan la implementación de proyectos de bioenergía para la generación de electricidad y las formas de coordinación de las transacciones que adoptan estos proyectos. El análisis se basa en la economía de los costos de transacción y en el estudio de cinco proyectos de generación de bioenergía para la red eléctrica desarrollados en la provincia de Buenos Aires, Argentina.

Palabras clave: Fuentes alternativas de energía, comportamiento organizacional, costos de transacción.

Clasificación JEL: Q42; D23

\section{Abstract}

Even though there are important opportunities for the development of the bioenergy industry in Latin America, this industry is lagging regarding electricity generation. The international literature agrees the industry faces important economic, social, and institutional barriers to penetrate the electric market. To address these barriers, individual bioenergy projects develop several forms of coordination of the resources available throughout the production chain. This study presents the results of an exploratory research on the barriers which condition the implementation of bioenergy projects and the organizational forms they adopt to coordinate resources. The analysis is based on Transaction Cost Economics and the study of five bioenergy projects for grid supply develop in Buenos Aires Province, Argentina.

Keywords: Alternative energy source, organizational behavior; transaction costs.

Classification JEL: Q42, D23 


\section{Introducción}

Existen importantes oportunidades para el desarrollo de la industria de la bioenergía en América Latina (AL) por la diversidad y amplia disponibilidad de biomasa ${ }^{1}$ y de tierras aptas para el uso agrícola sin poner en peligro zonas forestales o áreas protegidas. Aunque la evidencia muestra que la producción de bioenergía, especialmente de biocombustibles, puede incidir negativamente en la seguridad alimentaria por medio de la competencia en el uso de las tierras y, consecuentemente, en el precio de los alimentos (De Freitas Vian, De Moraes y Schneider Braun, 2013), con planificación política, esta industria puede contribuir a abordar desafíos claves para el desarrollo de los países de AL, como la seguridad energética, la emisión de gases de efecto invernadero, las condiciones ambientales de producción y hábitat y el desarrollo rural. Esta forma de energía plantea la instalación de nuevas cadenas de valor y mercados, una nueva forma de articulación a nivel regional entre la producción primaria y la producción industrial, y, de esta forma, la redefinición de los centros de producción desde las áreas urbanas a las rurales (Henry, Pahun y Trigo, 2014).

La bioenergía representa alrededor del 10\% de la capacidad de generación de energía eléctrica de fuentes renovables en AL. Aunque en los últimos cinco años el aumento de esta capacidad fue impulsado por las energías hidroeléctrica y eólica, la bioenergía creció en términos absolutos desde el año 2000. Sin embargo, este dinamismo se debe principalmente a proyectos desarrollados en Brasil y en los sectores azucarero y forestal (Irena, 2016).

En Argentina, la industria de la bioenergía se encuentra sumamente rezagada en materia de generación de energía eléctrica: el 0,18 \% de la demanda del Mercado Eléctrico Mayorista es cubierta con bioenergía proveniente de biogás, biomasa (incineración) y biodiésel (CAMMESA, 2016). En el año 2016, el Gobierno nacional licitó 1000 MW de potencia de energía eléctrica de fuentes renovables; se presentaron 11 proyectos de bioenergía (en los segmentos biomasa y biogás), de los cuales 8 superaron la revisión técnica, pero solo uno presentó una oferta económica por debajo del precio tope establecido por el Ministerio de Energía y Minería para el segmento de biogás (160 USD/ $\mathrm{MWh})^{2}$. Aunque esta licitación expuso el potencial de biomasa que existe en el país,

1 La industria de la bioenergía comprende la producción de combustibles líquidos, como el biodiésel y el bioetanol, y la producción de energía térmica y/o eléctrica a partir de tres tecnologías, de acuerdo con el tipo de biomasa: biodigestión, gasificación e incineración. La biomasa, por otra parte, denomina a toda materia orgánica originada en un proceso biológico accesible como fuente de energía e incluye tanto cultivos energéticos como biomasa de origen residual. Los cultivos energéticos son plantaciones de crecimiento rápido realizadas con el propósito específico de producir energía. La biomasa residual está compuesta por subproductos o residuos orgánicos generados en actividades agrícolas, silvícolas y ganaderas y en las industrias asociadas con estos sectores.

2 Frente a los reclamos informales del sector privado por la inadecuación de los pliegos de licitación nacional a las condiciones de los proyectos de bioenergía, el Ministerio de Energía y Minería adjudicó los 7 proyectos de biogás y biomasa que habían superado la revisión técnica bajo la condición de que los oferentes adecuaran el precio de oferta 
también mostró que la industria de la bioenergía es aún incipiente y que no existen cadenas de producción estructuradas, sino más bien actores con intereses dispersos y mercados poco transparentes.

En los países de la Unión Europea y en Estados Unidos, donde la industria de la bioenergía se encuentra más desarrollada, la literatura coincide en destacar la importancia de barreras económicas, sociales e institucionales en la penetración de esta industria en el mercado de la energía eléctrica (Altman y Johnson, 2008; Mayfield, Foster, Smith, Gan y Fox, 2007; McCormick y Kåberger, 2007; Roos, Graham, Hektor y Rakos, 1999; Rösch y Kaltschmitt, 1999; Thornley y Prins, 2009). Algunas de las barreras más significativas son aspectos que inciden en la complejidad y en la eficiencia de las cadenas de producción de bioenergía, incluyendo los costos logísticos de la biomasa (Adams, Hammond, McManus y Mezzullo, 2011; Blumer, Stauffacher, Lang, Hayashi y Uchida, 2013; McCormick y Kåberger, 2007); la baja integración y/o cooperación de los distintos sectores involucrados (Mayfield et al., 2007; McCormick y Kåberger, 2007; Roos et al., 1999); la competencia por la materia prima con mercados no energéticos (Adams et al., 2011; Smith, Lattimore, Berndes, Bentsen, Dimitriou, Langeveld y Thiffault , 2015); las dificultades asociadas con la producción y la movilización de recursos materiales, cognitivos y relacionales a nivel local (Adams et al., 2011; Rösch y Kaltschmitt, 1999); la incertidumbre del entorno, el poco conocimiento y la mala percepción pública de estas tecnologías (Hodgson, Ruiz-Molina, Marazza, Pogrebnyakova, Burns, Higson, Rehberger, Hiete, Gyalai-Korpos, Di Lucia, Noël, Woods y Gallagher , 2016; Mangoyana y Smith, 2011).

El abordaje de estas barreras mediante acciones públicas y privadas consensuadas entre los distintos actores que componen la industria de la bioenergía es un proceso necesario para promover el dinamismo y la sustentabilidad de sus cadenas de producción y el crecimiento de esta industria ${ }^{3}$. A nivel individual, los proyectos de bioenergía desarrollan diversas formas de organización que promueven el compromiso de las partes y facilitan la producción, la movilización y el acceso a recursos -información, organización, infraestructura, dinero, personal, apoyo político, etcétera- necesarios para implementar cadenas de producción de bioenergía sostenibles, adaptadas a las condiciones específicas de su entorno (Gold y Seuring, 2011; McCormick y Kåberger, 2007) y lo suficientemente robustas y flexibles como para afrontar la incertidumbre de los mercados (Iakovou, Karagiannidis, Vlachos, Toka y Malamakis, 2010).

Este trabajo presenta los resultados de una investigación exploratoria sobre las barreras que condicionan la implementación de proyectos de bioenergía para la generación de

al menor valor entre el precio ofertado y el precio máximo de adjudicación (160 USD/MWh para biogás y 110 USD/MWh para biomasa).

3 Para mayor detalle sobre las acciones necesarias para movilizar las cadenas de producción de bioenergía, se puede consultar el reporte Mobilizing sustainable bioenergy supply Chains, editado por IEA Bioenergy (Smith et al., 2015). 
electricidad y las formas de organización que adoptan estos proyectos para superarlas. El foco de este trabajo se halla en las cadenas de producción de bioenergía y su relación con el territorio, más que en el entorno económico e institucional de estos proyectos a nivel nacional. El análisis se basa en la aplicación de conceptos estándares de la economía de los costos de transacción (ECT) y en el estudio de cinco proyectos de generación de bioenergía para la red eléctrica desarrollados en la provincia de Buenos Aires, Argentina, por cooperativas de eléctricidad y empresas de capital individual del sector agropecuario y agroindustrial.

La siguiente sección desarrolla brevemente el enfoque de la ECT. La tercera parte presenta el contexto en el que surgen los proyectos analizados y la metodología utilizada. En el cuarto acápite se describen las barreras que enfrentan estos proyectos y las formas de organización que adoptan las empresas que integran la cadena de producción para reducir la incidencia negativa de estos factores. En la última sección se mencionan algunos aprendizajes que deja este estudio sobre las cadenas de producción de bioenergía y las barreras que estos proyectos enfrentan para su implementación, así como su impacto en la industria.

\section{Las cadenas de producción de bioenergía en la ECT}

Las cadenas de producción son estructuras dinámicas en las que participan organizaciones legalmente separadas, vinculadas entre sí por un flujo constante de recursos materiales, cognitivos y relacionales que les permiten alcanzar un objetivo común (Chopra y Meindl, 2008). En la industria de la bioenergía, estas cadenas están integradas esencialmente por empresas que producen materia orgánica, la transforman en un insumo energético técnicamente accesible y económicamente eficiente -mediante su densificación energética, almacenamiento y/o transporte- y la convierten en energía eléctrica y/o térmica (Iakovou et al., 2010; Rentizelas, Tolis y Tatsiopoulos, 2009).

Figura 1. Principales etapas en las cadenas de producción de bioenergía

Producción y/o recolección de materia orgánica
Densificación, almacenamiento y/o transporte de biomasa

Fuente: elaboración propia.
Generación de energía eléctrica y/o térmica

En cada una de estas etapas, las cadenas de producción adoptan distintas formas de coordinación de sus recursos, que responden, en términos de la ECT (Williamson, 1973 
y 1985), al interés de las empresas por disminuir los costos de transacción ${ }^{4}$. Este enfoque postula a la empresa (integración vertical, propiedad unificada) y al mercado (separación, propiedad dispersa) como dos formas extremas de organizar la transacción de recursos en un continuo de modalidades intermedias que combinan principios y reglas propias de cada una (Rialp Criado, 1998) . Estas formas intermedias incluyen acuerdos de cooperación heterogéneos por medio de los cuales las partes preservan su autonomía, pero aceptan obligaciones y ofrecen garantías de manera voluntaria respecto a su conducta presente y futura.

Los acuerdos de cooperación pueden ser clasificados en tres grandes categorías: 1) acuerdos de intercambio (próximos al mecanismo de mercado); 2) participaciones accionarias minoritarias, consorcios y alianzas entre empresas; y 3) joint ventures o empresas conjuntas (próximos a la propiedad unificada) (Rialp Criado, 1998). Las dos últimas categorías, en particular, presentan tres características analíticamente diferenciables: relaciones de contratación que implican vínculos de largo plazo, apoyados mayormente en la confianza; intercambios de información formales e informales y aprendizajes conjuntos, que permiten reducir la brecha cognitiva entre las partes y así alcanzar buenos niveles de comunicación; y acción colectiva en diferentes espacios, como mecanismo para promover la adopción de comportamientos cooperativos (Knorringa y Meyer-Stamer, 1998).

En el marco de la ECT, la elección de estas formas de coordinación depende de 1) los atributos de la transacción; 2) el comportamiento de los agentes involucrados en la transacción; y 3) el ambiente institucional que rodea la interacción entre las organizaciones (Williamson, 1985). Las transacciones, a su vez, pueden ser caracterizadas a partir de tres atributos: la frecuencia, la especificidad de los recursos involucrados y la incertidumbre. La frecuencia se refiere a la recurrencia de la transacción y al período a lo largo del cual se prolonga el intercambio. Ambas variables inciden en el costo de cada transacción, ya que la elaboración y el seguimiento de contratos específicos, por ejemplo, implican costos medios elevados si no se justifican en transacciones recurrentes (Williamson, 1985).

La especificidad de los activos se refiere a la movilidad relativa entre usos alternativos de aquellos recursos durables adquiridos específicamente para lograr determinadas

4 Para algunos autores, la eficiencia económica no es suficiente para explicar la modalidad de gobierno que adoptan las empresas. Powell (1990) sugiere que las organizaciones en red -como denomina el autor a “...una colección de actores $(\mathrm{N}>=2)$ que aspiran a relaciones de intercambio perdurables y repetitivas entre ellos y, al mismo tiempo, carecen de una autoridad organizativa que arbitre y resuelva las dispuestas que puedan surgir durante el intercambio" (Podolny y Page, 1998, p. 59)- ofrecen beneficios concretos o activos intangibles más valiosos, como la disminución de la incertidumbre, el acceso a información, la confiabilidad y la capacidad de respuesta, etc. También en esta línea, Nooteboom (2001) destaca la incertidumbre del entorno y la falta de acceso a información especializada como factores que promueven formas de coordinación diferentes al mercado para adquirir conocimientos complementarios.

5 Esta idea de continuidad entre las formas de coordinación de la actividad económica, sin embargo, es considerada por Powell (1990) como una visión estática e históricamente inadecuada, que limita la posibilidad de analizar otras formas viables de coordinación, como la colaboración y la reciprocidad. 
transacciones (García Garnica, Lara Rivero y Taboada Ibarra, 2004). Cuando el grado de especificidad de los activos es alto, las transacciones no son ni estandarizadas ni impersonales, sino que existe una dependencia bilateral que motiva el desarrollo de medidas contractuales y organizacionales para reducir los riesgos de la transacción, entre ellos, la posibilidad del oportunismo (Williamson, 1985).

La especificidad de los activos puede asumir, al menos, cuatro tipologías diferentes según Williamson (1985): especificidad de sitio, física, de capital humano y de activos dedicados. La especificidad de sitio se asocia con transacciones que deben realizarse en lugares definidos porque de lo contrario el activo perdería su valor.

La especificidad física tiene que ver con características de diseño de los activos que podrían condicionar su uso y, por lo tanto, su valor en una actividad alternativa. Esto ocurre con aquellos activos diseñados a medida o para usos sumamente específicos. Los sistemas de generación de bioenergía son susceptibles de tener activos con un alto grado de especificidad física, ya que, aun cuando son diversas las tecnologías disponibles para convertir la biomasa en energía, a medida que esta se especializa, el nivel de eficiencia de la planta de conversión depende cada vez más de la calidad y del tipo de biomasa (Altman y Johnson, 2008; Shelanski y Klein, 1995). En este sentido, el nivel de especificidad de las plantas de generación de energía depende de la flexibilidad de su tecnología con respecto a la calidad y al tipo de biomasa.

La especificidad de capital humano se relaciona con el conocimiento acumulado por los empresarios y trabajadores de una empresa y cuya aplicabilidad en funciones alternativas es limitada. En la industria de la bioenergía, la especificidad de los activos humanos se observa en los productores agrícolas, especialmente si estos deben aprender sobre la producción de nuevos cultivos y sus técnicas de acondicionamiento (como el secado), y en quienes mantienen y operan los equipos de transformación de la biomasa.

Por último, la especificidad de los activos dedicados se asocia con inversiones destinadas a satisfacer las necesidades de un socio comercial específico, lo que implica un alto nivel dependencia del cliente. La mayoría de las inversiones necesarias para que la industria de la bioenergía tome forma puede ser caracterizada como dedicada, ya que ni los productores de biomasa estarán dispuestos a adaptar su sistema de gestión de residuos o a dedicar recursos físicos y humanos a producir cultivos energéticos, ni los generadores estarán dispuestos a invertir en una planta de energía para un tipo específico de biomasa sin la seguridad de que cada uno cumplirá su parte de la inversión (Choinière, 2002).

La incertidumbre es otro atributo del intercambio que adquiere especial importancia cuando los activos son específicos y los agentes poseen una racionalidad limitada. La incertidumbre describe cambios en el entorno que obligan a los agentes a adaptarse a circunstancias espacio-temporales, pero Williamson (1985) también utiliza este término 
para referirse a la percepción de los agentes respecto a los cambios en el ambiente. $\mathrm{Si}$ los activos que apoyan la transacción tienen un alto nivel de especificidad, entonces, en condiciones de incertidumbre, las partes deberán contar con estrategias organizacionales que les permitan adaptarse a los cambios del entorno y mantener los flujos de intercambio. Las cadenas de producción de bioenergía enfrentan varias fuentes de incertidumbre: desde la variabilidad del precio de los combustibles sólidos y de otros bienes asociados hasta la posibilidad de contingencias climáticas que incidan en la producción de biomasa.

En cuanto al comportamiento de los agentes involucrados en la transacción, la ECT supone que poseen una racionalidad limitada, que tienen limitaciones neurofisiológicas y de lenguaje que limitan su capacidad de recibir, almacenar y procesar información correctamente y de expresar sus conocimientos y sentimientos mediante palabras y símbolos comprensibles (Arámbula y Gómez Álvarez, 1993). Como corolario de este supuesto, la ECT plantea la posibilidad de que las partes desarrollen acciones que distorsionen $\mathrm{u}$ oculten información relevante con el objetivo de alcanzar ventajas transaccionales. Como no es posible evaluar la probabilidad de ocurrencia de este comportamiento oportunista, la ECT considera que esto siempre puede ocurrir.

Sin embargo, las transacciones económicas están condicionadas por la confianza, y esta, a su vez, se fortalece cuando las relaciones de cooperación son exitosas, disminuyendo la probabilidad de comportamientos oportunistas (Nooteboom, 2001). La falta de confianza, por el contrario, responde a la distancia cognitiva de las partes, producto de conocimientos y experiencias diferentes y a la ausencia de un lenguaje común (García Garnica et al., 2004). Para superar la falta de confianza, las partes deben desarrollar un conjunto compartido de categorías y un lenguaje, al menos parcialmente propio de su relación, lo que puede demandar inversiones específicas. Esto implica un proceso de aprendizaje e innovación dinámico que la EGT no logra abordar (Nooteboom, 2001).

En el marco de la ECT, la interacción entre la incertidumbre y la racionalidad limitada explica que los agentes no sean capaces de prever todas las contingencias que pueden afectar una transacción y que, por lo tanto, desarrollen contratos incompletos, regímenes contractuales elásticos (con mecanismos de adaptación a perturbaciones imprevistas) o contratos que actúan como marco de acción o guía de consulta (Williamson, 1991). En las relaciones de largo plazo, la probabilidad de que existan comportamientos oportunistas disminuye motivada por la reputación o el prestigio de las empresas y sus consecuencias sobre los acuerdos informales que complementan los contratos (Taboada Ibarra y García Garnica, 2012).

Por último, el ámbito institucional se refiere al conjunto de reglas políticas, sociales y legales que enmarcan las actividades de producción, intercambio y distribución. Estas reglas estructuran y regulan el comportamiento social y se integran por costumbres, 
valores, rutinas, estándares, lenguajes, pensamientos, ritos y creencias, procesos sociales e históricos que rigen la forma de vida de los individuos, las empresas y la sociedad en su conjunto. Algunas reglas forman parte del marco regulatorio (son exógenas a las organizaciones) de las relaciones contractuales (Nooteboom, 2009). Otras son convenciones sociales (Gyau y Spiller, 2008) que involucran esquemas de sanción que se internalizan y se anticipan al comportamiento de las partes, desanimando el oportunismo. De esta forma, las normas sociales promueven la reputación de las partes y procesos de aprendizaje que facilitan su retroalimentación y aumentan su colaboración (su solidaridad) (García Garnica et al., 2004).

\section{Contexto del estudio y metodología}

Desde la década de los noventa, las cooperativas de electricidad en Argentina han desarrollado su actividad económica en un contexto adverso a su sostenibilidad. Con un mercado de electricidad altamente regulado, restricciones en el abastecimiento interno y tarifas de distribución a usuarios finales congeladas en un contexto inflacionario, estas entidades han enfrentado dificultades financieras y la disminución continua de la calidad de su servicio. Estas condiciones han impulsado a las cooperativas a explorar estrategias competitivas para diversificar su oferta de productos, retomar su función de generadoras de energía eléctrica, mejorar la calidad de su servicio y contribuir a abordar los desafíos ambientales de sus comunidades.

En respuesta a la ausencia de políticas energéticas que promovieran sistemas de generación de energía distribuida en el campo de las energías renovables, el Foro Regional Eléctrico de la provincia de Buenos Aires (FREBA) creó en el año 2009 el Programa Provincial de Incentivos a la Generación de Energía Distribuida (PROINGED) en el ámbito de la Secretaría de Servicios Públicos del gobierno de la provincia de Buenos Aires (PBA), una de las provincias más extensas y ricas del territorio argentino. El programa realiza actividades de investigación y desarrollo y financia estudios de preinversión y la ejecución de proyectos de energías renovables mediante préstamos a 10 años, a una tasa de mercado pasiva y variable en función de las colocaciones financieras de sus fondos. Así, el PROINGED financia proyectos en los que participan empresas distribuidoras de energía eléctrica en la red que son miembros de FREBA.

FREBA es una asociación civil sin fines de lucro que agrupa a empresas distribuidoras de electricidad de la provincia y a dos empresas de transporte de electricidad como miembros asesores. La asociación se encuentra integrada por 206 cooperativas de electricidad que brindan el servicio al $20 \%$ de los usuarios de la PBA y 4 empresas privadas que atienden el resto de los mercados (Ministerio de Energía y Minería, 2014). 
La PBA posee las tierras más fértiles del país y una vasta tradición en la actividad agropecuaria. Si bien el sector primario representa alrededor del $4 \%$ del producto bruto geográfico provincial, su encadenamiento productivo con otros sectores es muy relevante por la demanda de productos químicos, maquinaria agrícola y tecnología y su oferta de materia prima a la industria. Además, los sectores agropecuario y agroindustrial de esta jurisdicción aportan una porción importante de las exportaciones provinciales y nacionales (Ministerio de Economía - Dirección Provincial de Estudios y Proyecciones Económicas, 2012).

En línea con esta realidad, la mayoría de los proyectos financiados por el PROINGED no solo involucra a cooperativas eléctricas (12 de 18), sino que además propone la generación de energía eléctrica a partir de biomasa (11 de 18) (tabla 1). Estos proyectos representan una muestra de una población incierta, pero probablemente muy pequeña, de proyectos de generación de bioenergía para la red eléctrica en la PBA que han superado la etapa de diseño en el país ${ }^{6}$.

El estudio analiza 5 de los 8 proyectos de generación de energía eléctrica a partir de biomasa financiados por el PROINGED en los que participan cooperativas de electricidad. En todos los casos, se trata de proyectos destinados a la generación de energía eléctrica para su comercialización en la red, en los que participan cooperativas de electricidad en las etapas de generación y distribución de la energía. El estudio de estos casos en el marco de la ECT permite explorar las barreras sociales, económicas e institucionales que condicionan la implementación de estos proyectos y las formas de organización diseñadas para superarlas de manera sostenible.

Tabla 1. Proyectos financiados por PROINGED según fuente de energía y tipo de organización (2016)

\begin{tabular}{|l|l|c|c|}
\hline & Fuente de energía & $\begin{array}{c}\text { Entidades } \\
\text { cooperativas }\end{array}$ & $\begin{array}{c}\text { Entidades no } \\
\text { cooperativas }\end{array}$ \\
\hline \multirow{2}{*}{ Biomasa } & Número de proyectos & 8 & 3 \\
\hline \multirow{2}{*}{ Hidroeléctrica } & MW & 26,9 & 7,5 \\
\hline & Número de proyectos & 1 & 1 \\
\hline \multirow{2}{*}{ Solar } & Número de proyectos & 0,80 & 0,80 \\
\hline & MW & 0 & 2 \\
\hline
\end{tabular}

6 En septiembre de 2015, el Programa Probiomasa, del Gobierno nacional, tenía registro de 27 proyectos de bioenergía distribuidos en todo el país, 5 de los cuales se encontraban en la PBA (Energía Estratégica, 2015). En la licitación de potencia para energía renovable que realizó el Gobierno nacional en septiembre de 2016, se presentaron solo 8 proyectos de bioenergía, ninguno de los cuales estaba localizado en esta provincia. 


\begin{tabular}{|l|l|c|c|}
\hline & Fuente de energía & $\begin{array}{c}\text { Entidades } \\
\text { cooperativas }\end{array}$ & $\begin{array}{c}\text { Entidades no } \\
\text { cooperativas }\end{array}$ \\
\hline Eólica & Número de proyectos & 3 & 0 \\
\hline Total & MW & 4,75 & 0 \\
\hline & Número de proyectos & 12 & 6 \\
\hline
\end{tabular}

Fuente: elaboración propia sobre datos de PROINGED (2016).

La información utilizada en este trabajo fue recolectada mediante una combinación de entrevistas semiestructuradas personales y telefónicas con referentes de las cooperativas de electricidad y de empresas de capital individual involucradas en los proyectos, representantes del segmento de las energías renovables y funcionarios públicos nacionales y provinciales, e información proveniente de fuentes secundarias.

\section{Análisis de los sistemas de bioenergía y su forma de coordinación}

Los proyectos analizados en este trabajo se insertan en cadenas de producción de bioenergía formadas por cooperativas de electricidad y empresas de propiedad individual del sector agropecuario y agroindustrial. La participación de las primeras en los proyectos es un requisito del PROINGED, pero su participación en la etapa de distribución de energía eléctrica es una condición derivada de las regulaciones que rigen el mercado eléctrico nacional. A diferencia de otro tipo de empresas, las cooperativas son entidades con un fuerte anclaje territorial y con capacidad para promover la actividad económica y el empleo en su área de influencia. En este sentido, las cooperativas son, por lo general, motores de desarrollo local y referentes en la comunidad, y cuentan con una buena reputación económica y social (Mozas-Moral y Bernal-Jurado, 2006). Las empresas del sector agropecuario y agroindustrial, por otra parte, conforman el primer eslabón en la cadena de producción de bioenergía, ya que generan distintos tipos de biomasa de origen residual o cultivos energéticos. Estas empresas son clientes de las cooperativas de electricidad, y en los casos en que su consumo de energía eléctrica es alto, son categorizadas como grandes usuarios.

Los proyectos estudiados en este trabajo proponen la aplicación de diferentes tecnologías -incineración, biodigestión y el uso de biocombustibles líquidos-, utilizan diferentes tipos de biomasa - mayormente de origen residual-y, a comienzos del año 2016, transitaban distintas etapas (tabla 2); la mayoría había finalizado el análisis de factibilidad y se encontraba a la espera de que los organismos de control independientes (universidades 
nacionales) y el Estado nacional aprobaran el proyecto, y que el Ministerio de Energía y Minería fijara un precio de compra para la energía ofertada. El proyecto CYS, sin embargo, se encontraba en la etapa de desarrollo, a la espera de la llegada de dos generadores de biodiésel importados, mientras que el proyecto CT estaba en funcionamiento.

Tabla 2. Caracterización de los proyectos

\begin{tabular}{|c|c|c|c|c|}
\hline Denominación & Tipo de biomasa & $\begin{array}{c}\text { Tecnología } \\
\text { aplicada }\end{array}$ & $\begin{array}{c}\text { Potencia } \\
\text { (MW) }\end{array}$ & Etapa de desarrollo \\
\hline CT & Excretas vacunas & Biodigestión & 0,07 & En operación* \\
\hline CSAL & $\begin{array}{l}\text { Excretas de cerdos } \\
\text { y vacunos }\end{array}$ & Biodigestión & 1,4 & Análisis de factibilidad \\
\hline GRAL & Cama de pollo & Incineración & 6,0 & Análisis de factibilidad \\
\hline CFER & Chala y marlo & Incineración & 6,0 & Análisis de factibilidad \\
\hline CYS & Biocombustible líquido & Combustión & 5,0 & Desarrollo del proyecto \\
\hline
\end{tabular}

El proyecto CT fue impulsado por un productor agropecuario mediano que buscaba mejorar la rentabilidad de sus campos utilizando el fertilizante derivado de la biodigestión anaeróbica del estiércol y el efluente líquido de su ganado. Sin embargo, su motivación inicial fue superada por la posibilidad de obtener beneficios más elevados y generar electricidad a partir de biogás. La participación de la cooperativa de electricidad en el proyecto fue impulsada por el PROINGED, dado su interés por financiar iniciativas pioneras en la producción de bioenergía en el país. La cooperativa de electricidad, sin embargo, presentó inicialmente cierta reticencia a formar parte del proyecto debido al desconocimiento de la tecnología y sus potencialidades, así como por la incertidumbre que enfrentan los miembros de un consejo consultivo por ser solidariamente responsables con sus bienes personales del crédito que toma una cooperativa.

En la etapa de recolección, para lograr mayor eficiencia en la cadena de producción de bioenergía, los efluentes líquidos y el estiércol deben recogerse diariamente. Esta condición demanda modificaciones en el sistema de crianza del ganado (pasar de un sistema extensivo de cría a uno parcialmente intensivo) e inversiones en corrales con piso de hormigón para lograr una limpieza y un traslado más eficiente de la materia orgánica hacia el biodigestor. Estas inversiones son activos dedicados con una alta incidencia en la eficiencia del sistema.

Los residuos de la ganadería intensiva, a su vez, poseen una baja densidad energética que torna económicamente inviable su traslado, lo que condiciona la instalación del biodigestor en las cercanías de los corrales y lo transforma en un activo con una alta 
especificidad de sitio. De esta forma, la transacción de biomasa entre el productor y el operador del biodigestor encargado de su transformación en biogás posee una frecuencia elevada y demanda activos con una alta especificidad dedicada en la etapa de recolección y una alta especificidad de sitio en la etapa de obtención de biogás.

Adicionalmente, la frecuencia de transacción del biogás para su conversión en energía es alta y sus costos de almacenamiento y transporte no justifican su transformación en energía eléctrica en un lugar alejado de la planta de biodigestión. Esto otorga especificidad de sitio a los equipos y a las maquinarias involucrados en la transacción. Por otra parte, como la distribución de electricidad al mercado nacional debe ser provista por la cooperativa, su participación en la etapa de generación de electricidad es estratégica para acceder a condiciones de compra de la energía eléctrica (contratos de largo plazo con el Estado nacional) que reduzcan la incertidumbre del mercado de las energías renovables a nivel nacional (posibles cambios en la normativa, de los precios relativos, de las condiciones del préstamo de PROINGED, etc.).

Como resultado de estas condiciones, en el proyecto CT, el biodigestor es propiedad del productor y se encuentra emplazado en su predio, mientras que el equipo de generación de electricidad a partir de biogás es de la cooperativa, pero también está instalado en el terreno del productor. De esta forma, mientras las etapas de recolección y transformación de la biomasa están integradas verticalmente y son gestionadas por el productor, la transacción de biogás entre el productor y la cooperativa se encuentra coordinada por un acuerdo de intercambio de largo plazo, con salvaguardas para disminuir la incertidumbre del mercado energético y evitar comportamientos oportunistas de parte del productor ${ }^{7}$.

El proyecto CSAL tiene su origen en dos empresas del sector agropecuario que por varios años exploraron la posibilidad de agregar valor a su cadena de producción generando biofertilizante y energía eléctrica a partir del efluente líquido y el estiércol de su ganado vacuno y porcino. Para la cooperativa de electricidad, este proyecto representó una oportunidad para mejorar la calidad de su servicio, que hoy se encuentra condicionado por episodios de baja tensión en la red.

Por las características de la biomasa que utiliza este proyecto, sus costos de transacción a lo largo de la cadena de producción de bioenergía son similares a los del proyecto CT, por lo que la dependencia bilateral entre las partes es sumamente alta. Sin embargo, el proyecto CSAL plantea una forma de coordinación que, aunque próxima a la integración vertical, adopta una forma intermedia. El proyecto propone la constitución de dos

7 El contrato tiene un plazo de 10 años y establece, entre otras cuestiones, el precio de venta de la energía eléctrica y que el precio de compra del biogás está fijado por el Estado nacional y condicionado a la venta de energía eléctrica a CAMMESA. Adicionalmente, como parte del acuerdo, el productor contrató un seguro de caución a favor de la cooperativa para que, en caso de que la planta de biodigestión no funcione, esta pueda devolver el préstamo adquirido para comprar el generador. 
empresas conjuntas bajo la forma legal de la sociedad anónima, cada una integrada por la cooperativa y una de las empresas agropecuarias. Las empresas conjuntas serán responsables de la transformación de la biomasa en biogás mediante biodigestores anaeróbicos y de su posterior conversión en energía eléctrica, la cual será distribuida por la cooperativa de electricidad. Este acuerdo de cooperación plantea un vínculo de largo plazo entre las organizaciones, con el objetivo de disminuir la incertidumbre del proyecto al delimitar el impacto de su desempeño económico al patrimonio aportado por cada una de las partes.

El proyecto GRAL propone utilizar cama de pollo para generar energía eléctrica a partir de su conversión termoquímica por incineración. La cama de pollo ${ }^{8}$ es un residuo de la producción avícola cuya adecuada disposición final representa un costo relevante para las empresas productoras de pollos. Tiene un bajo nivel de humedad y un poder calorífico relativamente alto, y su traslado fuera de los establecimientos para su disposición final es una práctica habitual del sector. A diferencia de los proyectos anteriores, su recolección no debe ser diaria -la cama de pollo se utiliza por períodos de entre 45 y 60 días- y puede ser almacenada con baja tecnología. En este sentido, su frecuencia de transacción es media. Además, los productores avícolas no necesitan realizar inversiones específicas para lograr una recolección eficiente.

Los costos logísticos son una variable con gran incidencia en la rentabilidad de los sistemas de bioenergía en general. La densificación de la materia orgánica es un proceso que permite mejorar la eficiencia en las etapas de transporte y almacenamiento al aumentar la densidad de la biomasa (secado, pelletización) y/o disminuir su volumen (fardos). Sin embargo, como no existen tecnologías técnicamente maduras para compactar este y otros tipos de materia orgánica, la distancia de transporte de la biomasa desde el establecimiento de origen a la planta de transformación no puede exceder los $70 \mathrm{~km}$. Adicionalmente, las normas ambientales de carácter local pueden incrementar los costos de transporte y almacenamiento si se exigen permisos o arreglos logísticos que condicionen la gestión de la biomasa, especialmente cuando se trata de residuos como la cama de pollo. Todos estos costos limitan el volumen de biomasa que puede adquirir una planta de generación de bioenergía de manera rentable y, por ende, su escala de producción, transformándola en un activo con cierta especificidad de sitio en la transacción de biomasa ${ }^{9}$.

La especificidad de sitio de la planta de generación de energía motivó la cooperación entre la empresa avícola y la cooperativa de electricidad, pero la ausencia de normas

8 La cama de pollo está compuesta por una mezcla de cáscara de arroz y aserrín, y luego de ser utilizado por los pollos contiene, además, guano y restos de alimento.

9 Sin embargo, esta planta no puede ser considerada un activo dedicado, ya que la tecnología que utiliza (incineración) le brinda flexibilidad para utilizar biomasa seca de otros establecimientos avícolas, forestales o de la poda urbana, por ejemplo, ubicados a no más de $70 \mathrm{~km}$ a la redonda. 
locales claras sobre el almacenamiento y la incineración de materia orgánica, así como la posible oposición de la opinión pública a esta práctica, estimuló el desarrollo de una relación de cooperación interorganizacional entre estas y el municipio. En este proyecto, estas relaciones se traducen en una alianza que se propone adoptar la forma legal de una unión transitoria de empresas, la cual será responsable de la gestión de los recursos necesarios para la transformación de la biomasa en energía eléctrica. Más cercana al mecanismo de mercado que la empresa conjunta, esta alianza tripartita establece un precio para la materia orgánica, deja abierta la posibilidad de recurrir a otros proveedores y tipos de biomasa para mantener o ampliar la generación de energía eléctrica y permite el diseño de un sistema de incentivos adecuado a los riesgos, intereses y recursos aportados por cada una de las partes.

El proyecto CFER fue desarrollado por una cooperativa de electricidad para generar energía mediante la conversión termoquímica por incineración de biomasa seca, principalmente chala y marlo. Aunque con una tecnología y un tipo de biomasa similar al proyecto anterior, este proyecto plantea una forma de organización de la cadena de producción próxima a la propiedad dispersa, apoyada en la expectativa de que el mercado de biomasa seca de origen residual se desarrolle en la región, impulsado por la actividad de la planta. Las condiciones que sostienen esta posibilidad son la abundante disponibilidad de chala y marlo en el área de influencia de la entidad y la ausencia de un destino adecuado para la disposición final de este residuo.

De acuerdo con las expectativas del proyecto, la oferta de biomasa seca podría llegar a superar la demanda y provenir de diversos productores agroindustriales de la zona dispuestos a ceder este subproducto a cambio de un certificado de disposición final de residuos. Sin embargo, como en el proyecto anterior, la planta de generación de bioenergía representa un activo con una alta especificidad de sitio debido a los costos logísticos, pues la rentabilidad de este proyecto depende de que la distancia de transporte de la biomasa no supere los $50 \mathrm{~km}$.

$\mathrm{El}$ almacenamiento de esta biomasa debe compatibilizar la oferta, disponible dos meses al año, con la demanda continua de este insumo que tiene la planta de bioenergía. Estas condiciones representan una baja frecuencia en cuanto a la transacción de biomasa, pero costos de almacenamiento elevados, dada la necesidad de controlar la calidad de la materia orgánica y evitar la pérdida de materia seca (Gold y Seuring, 2011).

Para reducir la incertidumbre del proyecto en sus primeras fases de operación, la cooperativa firmó un acuerdo de intercambio de chala y marlo con una de las principales empresas agroindustriales de la región. A diferencia de las formas de coordinación planteadas en los proyectos anteriores, este acuerdo de cooperación posee principios cercanos al mercado: las partes mantienen su autonomía, su relación se centra en la compra-venta de biomasa, se establece un precio de referencia para el insumo y no se 
ofrecen salvaguardas frente a contingencias en el entorno. A diferencia del mercado, sin embargo, la transacción entre las partes no es impersonal, ya que existe una relación comercial previa que define la reputación de estos agentes y su confianza.

Por último, en el proyecto GYS, la cooperativa de electricidad utiliza biodiésel para la generación por combustión de energía eléctrica, un biocombustible líquido derivado de aceites vegetales que posee un mercado nacional estable y estándares internacionales de calidad definidos. Por un lado, esta tecnología cuenta con una amplia flexibilidad para la sustitución de biodiésel por combustible diésel ante cambios presentes en las condiciones de los mercados. Por otro lado, en la PBA existen nueve pequeñas y medianas empresas productoras de este biocombustible, que generan alrededor del $8 \%$ de 4,6 millones de toneladas producidas a nivel nacional (CARBIO, 2015).

Este proyecto no enfrenta riesgos asociados con variaciones relacionadas con la calidad del insumo, la disponibilidad e incluso el transporte, por lo que la cooperativa no ha desarrollado acuerdos de cooperación con otros actores, adicionales a la organización del mercado, para llevar a cabo este proyecto. Esto se debe a la estabilidad y a la transparencia que el mercado de biocombustibles líquidos posee y a la flexibilidad de la tecnología utilizada para la generación de energía eléctrica.

La tabla 3 resume los atributos de las transacciones que constituyen la cadena de producción de bioenergía y las formas de coordinación adoptadas para disminuir los costos asociados con las características de cada sistema y las barreras del entorno.

Tabla 3. Resumen de los proyectos analizados según atributos de la transacción y formas de coordinación*

\begin{tabular}{|c|c|c|c|c|c|c|}
\hline \multicolumn{2}{|c|}{$\begin{array}{l}\text { Atributos de la } \\
\text { transacción }\end{array}$} & GT & GSAL & GRAL & GFER & GYS \\
\hline \multicolumn{2}{|c|}{$\begin{array}{l}\text { Frecuencia de la } \\
\text { transacción de biomasa }\end{array}$} & Alta & Alta & Media & Baja & A demanda \\
\hline \multirow{2}{*}{$\begin{array}{l}\text { Especificidad } \\
\text { de los activos }\end{array}$} & Dedicada & Alta & Alta & Baja & Baja & Nula \\
\hline & De sitio & Alta & Alta & Media & Media & Baja \\
\hline \multicolumn{2}{|c|}{ Formas de coordinación } & $\begin{array}{c}\text { Integración } \\
\text { vertical y } \\
\text { acuerdo de } \\
\text { intercambio con } \\
\text { salvaguardas }\end{array}$ & $\begin{array}{l}\text { Empresa } \\
\text { conjunta }\end{array}$ & Alianza & $\begin{array}{l}\text { Acuerdo de } \\
\text { intercambio } \\
\text { sin } \\
\text { salvaguardas }\end{array}$ & Mercado \\
\hline
\end{tabular}

Fuente: elaboración propia. * El uso de las categorías alta, media y baja es arbitrario y solo tiene sentido para comparar este grupo de proyectos. 


\section{Conclusiones}

Esta investigación, aunque exploratoria, brinda algunos aprendizajes sobre las cadenas de producción de bioenergía en Argentina, las barreras que condicionan a nivel proyecto su implementación y, por lo tanto, el desarrollo de esta industria y las formas de coordinación que adoptan las empresas para superar estas y otras dificultades.

Las cadenas de producción de bioenergía son sistemas complejos que requieren de la participación de actores con intereses disímiles; su configuración depende, en gran medida, del tipo de biomasa utilizada. Por un lado, el tipo de biomasa determina la frecuencia del intercambio y, dada la tecnología de densificación energética, el grado de especificidad de sitio de las plantas de generación de energía eléctrica. Sin embargo, cuando se trata de biomasa sólida, la tecnología aplicada a la generación de energía posee mayor flexibilidad para usar diferentes tipos de biomasa, lo que disminuye el nivel de especificidad dedicada de estos activos. De esta forma, como señala la teoría, a medida que los activos son menos específicos y la frecuencia de la transacción disminuye, la dependencia bilateral es menor, lo que se refleja en formas de coordinación más próximas al mercado.

Por otro lado, uno de los principales factores que condicionan la implementación de estos proyectos es la ausencia de mercados de biomasa. Los motivos son diversos: falta de control ambiental del sector público sobre el tratamiento de los residuos agropecuarios y agroindustriales, escasa información sobre el valor potencial de esta materia orgánica y altos costos de transporte derivados de la ausencia de tecnología de densificación adecuada y de infraestructura para su acondicionamiento y traslado. La ausencia de un mercado estable y transparente impone altos costos de transacción para la biomasa, independientemente de sus características físicas, ya que su intercambio demanda arduos procesos de negociación, la elaboración de contratos específicos y el despliegue de acciones para monitorear su cumplimiento. Estas acciones impregnan de desconfianza e incertidumbre las relaciones comerciales, aumentan la probabilidad de comportamientos oportunistas y condicionan la toma de decisiones estratégicas.

Frente a la complejidad de las cadenas de producción de bioenergía y la ausencia de mercados de biomasa, las empresas promotoras de los proyectos analizados plantean mayoritariamente formas de coordinación de propiedad unificada o acuerdos de cooperación relativamente próximos a la integración vertical y limitan la participación de otros agentes económicos, integrando las etapas de almacenamiento y acondicionamiento de la biomasa residual a la etapa de generación de energía eléctrica. Solo el proyecto CYS, con un mercado estable y transparente como el de los biocombustibles líquidos, recurre a una forma de coordinación descentralizada. 
Los acuerdos de cooperación que plantean estos proyectos se apoyan en relaciones de confianza construidas a lo largo de años de intercambio de energía eléctrica entre las cooperativas y las empresas. La confianza y la reputación facilitan la coordinación de múltiples recursos tangibles (dinero, infraestructura, personal capacitado, etc.) e intangibles (know-how, apoyo político, información, etc.), necesarios para la implementación de estos proyectos. De esta forma, los acuerdos de cooperación constituyen una solución organizacional a barreras que condicionan la sostenibilidad de los proyectos, al mismo tiempo que promueven la expansión de los mercados de biomasa a nivel local. Su importancia se torna aún más relevante cuando existen barreras de carácter institucional, como la ausencia de normativas precisas sobre la incineración de residuos orgánicos o la seguridad de las plantas de biodigestión, así como el desconocimiento entre los funcionarios públicos y políticos sobre la industria de la bioenergía y sus potencialidades.

Por último, es necesario profundizar esta línea de investigación para conocer cómo funcionan estas formas de coordinación y sus modelos de gobernanza en la etapa de operación de los proyectos. Para esto, será necesario complementar el enfoque de la ECT con conceptos provenientes de la sociología económica y el neoinstitucionalismo económico. Se espera que este análisis permita delinear propuestas de política pública alineadas con los intereses, los desafios y las soluciones de los propios actores que participan en las cadenas de producción de bioenergía.

\section{Referencias}

Adams, P. W., Hammond, G. P., McManus, M. C. y Mezzullo, W. G. (2011). Barriers to and drivers for UK bioenergy development. Renewable and Sustainable Energy Reviews, 15(2), 1217-1227. DOI: 10.1016/j.rser.2010.09.039

Altman, I. y Johnson, T. (2008). The choice of organizational form as a non-technical barrier to agro-bioenergy industry development. Biomass and Bioenergy, 32(1), 28-34. DOI: 10.1016/j.biombioe.2007.06.004

Arámbula, H. y Gómez Álvarez, D. (1993). La economía de los costos de transacción. Una explicación alternativa para el estudio de las organizaciones. Gestión y Politica Pública, II(2), 315-336. Recuperado de goo.gl/myRAl0

Blumer, Y. B., Stauffacher, M., Lang, D. J., Hayashi, K. y Uchida, S. (2013). Nontechnical success factors for bioenergy projects - Learning from a multiple case study in Japan. Energy Policy, 60, 386-395. DOI: 10.1016/j.enpol.2013.05.075

CAMMESA. (2016). Informe Anual 2015. Buenos Aires: Autor. Recuperado de goo. $\mathrm{gl} / 99 \mathrm{~b} 7 \mathrm{IU}$ 
CARBIO - Cámara Argentina de Biocombustibles. (2015). La producción de biodiésel en Argentina. Una decisión estratégica. Recuperado de goo.gl/m317j3

Choinière, C. J. (julio de 2002). Contract structure, learning-by-doing and the viability of new agricultural industries. Trabajo presentado en la Reunión Anual de la Asociación Americana de Economía Agrícola (American Agricultural Economics Association Annual Meeting), Log Beach, CA. Recuperado de goo.gl/t1Wdr6

Chopra, S. y Meindl, P. (2008). Administración de la cadena de suministro. Estrategia, planeación y operación (3.a ed) (Vol. 1). México: Pearson- Prentice Hall. Recuperado de goo. $\mathrm{gl} / 8 \mathrm{~B} 6 \mathrm{bDh}$

De Freitas Vian, G. E., De Moraes, G. I. y Schneider Braun, M. B. (2013). Políticas para relaciones contractuales en las cadenas de biocombustibles: construyendo puentes entre instituciones. Semestre Económico, 16(33), 15-44. Recuperado de goo.gl/yllb2k

Energía Estratégica. (29 de septiembre de 2015). Cuatro provincias lideran la generación de energía por biomasa en el país. Recuperado de goo.gl/wpU8MH

García Garnica, A., Lara Rivero, A. y Taboada Ibarra, E. (2004). La coordinación "híbrida" desde las perspectivas de Williamson y de Nooteboom. Revista Análisis Económico, XIX(401), 101-117. Recuperado de goo.gl/ZrYLYP

Gold, S. y Seuring, S. (2011). Supply chain and logistics issues of bio-energy production. Journal of Cleaner Production, 19(1), 32-42. DOI: 10.1016/j.jclepro.2010.08.009

Gyau, A. y Spiller, A. (2008). The impact of supply chain governance structures on the inter-firm relationship performance in agribusiness. Agricultural Economics, 54(4), 176 185. Recuperado de goo.gl/RuQTCL

Henry, G., Pahun, J. y Trigo, E. (2014). La bioeconomía en América Latina: oportunidades de desarrollo e implicaciones de política e investigación. FACES - Revista de la Facultad de Ciencias Económicas y Sociales, 20(42), 125-141.

Hodgson, E., Ruiz-Molina, M. E., Marazza, D., Pogrebnyakova, E., Burns, C., Higson, A., Rehberger, M., Hiete, M., Gyalai-Korpos, M., Di Lucia, L., Noël, Y., Woods, J. y Gallagher, J. (2016). Horizon scanning the European bio-based economy: A novel approach to the identification of barriers and key policy interventions from stakeholders in multiple sectors and regions. Biofuels, Bioproducts and Biorefining, 10(5), 508-522. DOI: $10.1002 /$ bbb. 1665 
Iakovou, E., Karagiannidis, A., Vlachos, D., Toka, A. y Malamakis, A. (2010). Waste biomass-to-energy supply chain management: A critical synthesis. Waste Management, 30(10), 1860-1870. DOI: 10.1016/j.wasman.2010.02.030

Irena - International Renewable Energy Agence. (2016). Renewables in Latin America and the Caribbean. Recuperado de goo.gl/BlmD6M

Knorringa, P. y Meyer-Stamer, J. (1998). New dimensions in local enterprise cooperation and Development: From clusters to industrial districs. En New approaches to science and technology co-operation and capacity building (Serie ATAS Bulletin XI). University of Duisburg, Alemania.

Mangoyana, R. B. y Smith, T. F. (2011). Decentralised bioenergy systems: A review of opportunities and threats. Energy Policy, 39(3), 1286-1295. DOI: 10.1016/j. enpol.2010.11.057

Mayfield, C. A., Foster, C. D., Smith, G. T., Gan, J. y Fox, S. (2007). Opportunities, barriers, and strategies for forest bioenergy and bio-based product development in the Southern United States. Biomass and Bioenergy, 31(9), 631-637. DOI: 10.1016/j. biombioe.2007.06.021

McCormick, K. y Kåberger, T. (2007). Key barriers for bioenergy in Europe: Economic conditions, know-how and institutional capacity, and supply chain coordination. Biomass and Bioenergy, 31(7), 443-452. DOI: 10.1016/j.biombioe.2007.01.008

Ministerio de Economía - Dirección Provincial de Estudios y Proyecciones Económicas. (2012). Panorama productivo de la provincia de Buenos Aires. Buenos Aires: Autor.

Ministerio de Energía y Minería. (2014). Informe Estadístico del Sector Eléctrico. Buenos Aires: Autor. Recuperado de goo.gl/duwblr

Mozas-Moral, A. y Bernal-Jurado, E. (2006). Desarrollo territorial y economía social. CIRIEG-España. Revista de Economía Pública, Social y Cooperativa, 55, 125-140. Recuperado de goo.gl/rM0krx

Nooteboom, B. (2001). Learning and governance in inter-firm relations (Documento de investigación, ERIM). Rotterdam: Erasmus Research Institute of Management - Erasmus University Rotterdam. Recuperado de goo.gl/RAzYGG

. (2009). A cognitive theory of the firm: learning, governance and dynamics capabilities. UK, Reino Unido: Edward Edgar Publishing.

Podolny, J. M. y Page, K. L. (1998). Network forms of organization. Annual Review of Sociology, 24, 57-76. DOI: 10.1146/annurev.soc.24.1.57 
Powell, W. (1990). Neither market nor hierarchy: Network forms of organization. Research in Organizational Behavior, 12, 295-336. Recuperado de goo.gl/Zxails

PROINGED - Programa Provincial de Incentivos a la Generación de Energía Distribuida (2016). Estudios y proyectos. Recuperado de proinged.com.ar

Rentizelas, A. A., Tolis, A. J. y Tatsiopoulos, I. P. (2009). Logistics issues of biomass: The storage problem and the multi-biomass supply chain. Renewable and Sustainable Energy Reviews, 13(4), 887-894. DOI: 10.1016/j.rser.2008.01.003

Rialp Criado, J. (1998). Selección de estructuras para materializar acuerdos de colaboración interempresarial. Moneda y Crédito, 206, 153-192.

Roos, A., Graham, R. L., Hektor, B. y Rakos, G. (1999). Critical factors to bioenergy implementation. Biomass and Bioenergy, 17(2), 113-126. DOI: 10.1016/ S0961-9534(99)00028-8

Rösch, C. y Kaltschmitt, M. (1999). Energy from biomass - Do non-technical barriers prevent an increased use? Biomass and Bioenergy, 16(5), 347-356. DOI: 10.1016/ S0961-9534(98)00088-9

Shelanski, H. A. y Klein, P. G. (1995). Empirical research in transaction cost economics : A review and assessment. Fournal of Law, Economics \& Organization, 11(2), 335-361. Recuperado de goo.gl/FR51XH

Smith, T., Lattimore, B., Berndes, G., Bentsen, N. S., Dimitriou, I., Langeveld, H. y Thiffault, E. (octubre de 2015). Mobilizing sustainable bioenergy supply chains. IEA Bioenergy Strategic Inter-Task Project. USA: IEA Bioenergy. Recuperado de goo.gl/xHYWR3

Taboada Ibarra, E. L. y García Garnica, A. (2012). Teoría de la empresa: las propuestas de Coase, Alchian y Demsetz, Williamson, Penrose y Nooteboom. Economía: Teoría y Práctica. Nueva Época, 36, 9-42. Recuperado de goo.gl/3CKYjK

Thornley, P. y Prins, W. (junio-julio de 2009). Barriers to European bioenergy expansion. Trabajo presentadao en la XVII European Biomass Conference and Exhibition: From Research to Industry and Markets, Hamburgo, Alemania. Recuperado de goo.gl/kMJFli

Williamson, O. E. (1973). Organizational forms and internal efficiency: Market and hierarchies: Some elementary considerations. American Economic Review, 63(2), 316-325. . (1985). The economic institutions of capitalism. Londres: Macmillan.

- (1991). Comparative economic organization: The analysis of discrete structural alternatives. Administrative Science Quarterly, 36(2), 269-296. 